\title{
An improved procedure for obtaining and maintaining well characterized partial water saturation states on concrete samples to be used for mass transport tests
}

\author{
Authors: Carlos Antón ${ }^{1}$, Miguel A. Climent ${ }^{1}$, Guillem de Vera ${ }^{1}$, Isidro Sánchez ${ }^{1}$, \\ Carmen Andrade ${ }^{2}$ \\ ${ }^{1}$ Departament d'Enginyeria de la Construcció, Obres Públiques i Infraestructura \\ Urbana, Universitat d'Alacant, PO Box 99, 03080 Alacant/Alicante, Spain. \\ ${ }^{2}$ Instituto de Ciencias de la Construcción Eduardo Torroja, CSIC, C/ Serrano \\ Galvache s/n, 28033 Madrid, Spain. \\ * Corresponding author. E-mail: ma.climent@ua.es
}

Tel: +34 965903400 ext. $2468 \quad$ Fax: +34 965903678.

\begin{abstract}
A conditioning procedure is proposed allowing to install into the concrete specimens any selected value of water saturation degree with homogeneous moisture distribution. This is achieved within the least time and the minimum alteration of the concrete specimens. The protocol has the following steps: obtaining basic drying data at $50^{\circ} \mathrm{C}$ (water absorption capacity and drying curves); unidirectional drying of the specimens at $50^{\circ} \mathrm{C}$ until reaching the target saturation degree values; redistribution phase in closed containers at $50^{\circ} \mathrm{C}$ (with measurement of the quasi-equilibrium relative humidities); storage into controlled environment chambers until and during mass transport tests, if necessary. A water transport model is used to derive transport parameters of the tested materials from the drying data, i.e. relative permeabilities and apparent water diffusion coefficients. The model also allows calculating moisture profiles during isothermal drying and redistribution phases, thus allowing optimization of the redistribution times for obtaining homogeneous moisture distributions.
\end{abstract}

Keywords: Concrete; Humidity; Water saturation degree; Conditioning;

Transport model. 


\section{Introduction}

It is generally accepted that concrete durability is to a large extent governed by concrete's resistance to the penetration of aggressive media, which may be present in a liquid or gaseous state (Hilsdorf 1995). The ingress and transport of aggressive species through concrete is governed by different physical-chemical mechanisms: diffusion, permeation, capillary suction and migration. Depending on the practical material and environmental conditions, the transport of media into concrete may be due to one or to several of the abovementioned mechanisms acting simultaneously. In the experimental investigation of the transport characteristics of concrete an attempt is normally made to limit the flow of media to one single transport mechanism in order to be able to derive reliable values of the transport coefficient corresponding to the particular mechanism (Kropp et al. 1995).

One of the conditions most influential on the transport properties of concrete is its moisture content and distribution, especially at the exposed surfaces of the specimens or structures (Ollivier et al. 1995). Gas permeability is significantly increased as moisture is removed from the capillary pores of concrete, due to the increase in the volume and connectivity of channels available for permeation (Parrott and Chen 1990; Romer 2005). Water absorption is also increased as the capillary pores of concrete are emptied of liquid (Nokken and Hooton 2002). These facts have led to the conclusion that test methods, for these last two properties, must include a standardized preconditioning, which must result in a controlled average moisture concentration with a uniform distribution (RILEM TC 116-PCD 1999a). The recommended preconditioning installs a defined intermediate moisture concentration in concrete, which is in equilibrium with the ambient air at $75 \%$ relative humidity $(R H)$ and a temperature of $20^{\circ} \mathrm{C}$. The preconditioning procedure consists of a predrying step at a temperature of $50^{\circ} \mathrm{C}$, followed by a redistribution phase also performed at $50^{\circ} \mathrm{C}$ (RILEM TC $116-\mathrm{PCD}$ 1999b). This last redistribution step was shown to be necessary for obtaining, within reasonable conditioning times, a uniform moisture distribution into the concrete specimens (Parrott 1994). Other preconditioning procedure has been proposed (Carcassès et al. 2002), which includes predrying and redistribution of 
moisture at $80^{\circ} \mathrm{C}$, and allows obtaining two levels of water saturation in concrete: 0.70 and 0.30 .

The pure mechanism of ionic diffusion is known to operate only when concrete is fully water saturated. It has been shown that when chloride $\left(\mathrm{Cl}^{-}\right)$diffusion tests are performed on non-saturated concrete specimens, or when the saturated specimens are allowed to dry during the test, the derived parameter values of the diffusion tests are significantly affected (McGrath and Hooton 1999; de Vera et al. 2007a). This is due to the fact that when moisture concentration gradients are present within a concrete specimen or structure, other mass transfer mechanisms different to diffusion, i.e. capillary absorption and wicking, start to contribute to chloride transport. A clearly different requirement is found here for the diffusion tests, as compared with gas permeability or capillary suction tests. For those latter techniques the goal of the preconditioning is to start the transport test at defined homogeneously distributed water contents, but for the former this water content and its homogeneity must be maintained throughout the diffusion test. For this reason great care is taken in natural ionic diffusion test procedures, to ensure both an initial full water saturation of concrete and an avoidance of the drying of the concrete surfaces (CEN/TC 104 2009).

Recently, several research groups have reported the experimental determination of the values of the $\mathrm{Cl}^{-}$diffusion coefficient under partial water saturation of concrete (Climent et al. 2000 and 2002; de Vera et al. 2000 and 2007b; Guimarães et al. 2000-2011; Nielsen and Geiker 2003). In all of these works care has been taken to work with mortar or concrete specimens preconditioned to several values of water saturation degree, to use a procedure for chloride deposition that does not alter the moisture content of the specimens and to avoid modifications of the water content within the concrete specimen during the diffusion test. Nevertheless, none of the abovementioned works has fully exploited all the available knowledge to design low time consuming preconditioning procedures that ensure to the greatest possible extent the installing and maintaining, during the whole diffusion test, of well characterized and homogeneously distributed water contents.

The aim of this work is to propose an improved experimental procedure for obtaining, (within reasonable conditioning times), and maintaining selected well characterized water contents, which can cover a sufficiently broad and significant 
range of saturation degrees of the concrete specimens. The protocol also allows determining the ambient $R H$ value in equilibrium with the preconditioned concrete specimens, in order to define the conditions ensuring the maintenance of the hygric state during the diffusion tests, (Antón 2009). The verification of homogeneity of the moisture distributions is performed by calculations through a mathematical model describing the water transfers between concrete and environment, with parameters obtained by fitting the model equations to experimental concrete drying tests data.

\section{Experimental}

For this study three concrete mixes, designated as $\mathrm{H} 1, \mathrm{H} 2$ and $\mathrm{H} 3$, were prepared using an ordinary Portland cement, type CEM I 42.5 R (MPR 2008), a 4 mm maximum size (fineness module: 3.2) limestone sand, two limestone gravels (4-6 and 6-12 $\mathrm{mm}$ respectively), distilled water, and a superplasticizer (modified polycarboxylate in aqueous solution). The concrete mixes, whose dosages are shown in Table 1, were designed to obtain medium compressive strengths at 28 days (30-45 $\mathrm{MPa})$, using water/cement (w/c) ratios between 0.38 and 0.60.

Two types of cylindrical specimens were cast: $30 \mathrm{~cm}$ in height and $15 \mathrm{~cm}$ in diameter for the compressive strength tests at 28 days, and $11-13 \mathrm{~cm}$ in height and $10 \mathrm{~cm}$ in diameter for conditioning and mass transport testing. After casting, the specimens were compacted by vibration. Next day of casting, the molds were removed and the specimens were cured by immersion in a saturated $\mathrm{Ca}(\mathrm{OH})_{2}$ solution during one year.

After curing, a water cooled diamond disc was used to slice specimens of $4 \mathrm{~cm}$ height for $\mathrm{H} 1$, and $3 \mathrm{~cm}$ height for $\mathrm{H} 2$ and $\mathrm{H} 3$, intended for the conditioning and mass transport tests. In all cases at least $15 \mathrm{~mm}$ of the bottom (contact with the mold base) and top ends of the $10 \mathrm{~cm}$ diameter cylinders were removed, before slicing two specimens from the central part of each cast concrete cylinder. A total number of 43 slices for each concrete type were used for this study.

The lateral surfaces of the cylindrical slices were sealed, before conditioning, in order to allow only unidirectional water vapour transfers. In this work vinyl electric isolating tape was used for the sealing. The $\mathrm{RH}$ measurements were performed with a Vaisala HM70 hygrometer. Laboratory temperature was maintained at $20^{\circ} \mathrm{C} \pm 2{ }^{\circ} \mathrm{C}$ throughout this study. 
Several properties of the concretes were measured in the fresh state: slump (AENOR 1990); and hardened state: compressive strengths at 28 days (AENOR 1984), and specific gravities and porosities before conditioning (ASTM 1994).

\section{Proposed conditioning procedure for obtaining well characterized partial water saturation states on concrete}

The objective of the proposed conditioning protocol is to allow obtaining, within the least time and with the minimum alteration of the microstructure of the concrete specimens, well characterized water saturation states with homogeneous moisture distributions. The starting point is the water saturated condition of concrete, and the partial saturation states are obtained by drying. The water saturation degree, $S D$, of concrete is defined as:

$$
S D=\frac{m_{h}-m_{d}}{m_{s}-m_{d}}(1)
$$

Where $m_{h}$ is the mass of the concrete specimen conditioned to the particular saturation state, $m_{d}$ is the mass of the dried specimen at $105^{\circ} \mathrm{C}$, and $m_{s}$ is the mass of the water saturated specimen. In this work the water saturated state is considered to be that obtained under vacuum (ASTM 1997), since this technique has been found to be the most efficient saturation procedure (Safiuddin and Hearn 2005). Nevertheless, the procedure can be applied considering other different saturation states (Nielsen and Geiker 2003).

The proposed procedure includes drying and water redistribution steps at $50^{\circ} \mathrm{C}$, in order to reduce time consumption. This leads to some requirements regarding the concrete specimens: the geometry and dimensions of the specimens must be kept constant for all the tests, since different geometries would imply different drying times for the same $S D$ value. The water vapour exchanges during drying must be unidirectional, in order to avoid tri-dimensional water content gradients, which are more difficult to eliminate during the redistribution steps (RILEM TC 116-PCD 1999b; Carcassès 2002). The application of relatively high temperature $\left(50^{\circ} \mathrm{C}\right)$ may modify the hydration degree of the cementitious binders. For this latter reason it is advisable to perform the pre-conditioning and the diffusion tests on highly matured concrete specimens, for instance cured under water for sufficiently 
long periods. This last precaution is particularly pertinent when supplementary cementing materials or blended cements are under study.

\subsection{Obtaining the drying curves and the water absorption coefficient}

The quantification of the mass loss, by drying from the saturated state, to achieve a certain value of $S D$ for a concrete specimen, requires knowing its dried mass. Nevertheless, several authors have indicated that drying at $105^{\circ} \mathrm{C}$ can produce irreversible microstructural modifications of the hardened cementitious matrices (Nokken and Hooton 2002; Safiuddin and Hearn 2005). This severe drying step can be avoided if the water absorption coefficient, $A$, is known.

$$
A=\frac{m_{s}-m_{d}}{m_{d}}
$$

By combining Equations (1) and (2) it is possible to calculate the mass of the concrete specimen, $m_{h}$, to be reached by drying from the saturated state, to reach the target $S D$ value.

$$
m_{h}=m_{s} \frac{1+S D \cdot A}{1+A}
$$

For this reason in this proposal, several concrete specimens, (at least four), different from the specimens intended for the transport tests, are used for obtaining the value of $A$ for the material under study, together with the drying curves. These latter are necessary for obtaining approximate values of the drying times needed to reach the desired $S D$ values.

The selected concrete specimens are first vacuum saturated, recording their $m_{s}$ values. The lateral surfaces are sealed in order to allow only unidirectional water vapour transfers, and the specimens are introduced in a ventilated oven at $50^{\circ} \mathrm{C}$. The decreasing mass of the specimens, $m_{h}$, is recorded as a function of time. The duration of this treatment must be adjusted to cover the whole $S D$ range to be studied. In this work the chosen set of saturation degrees was the following: 0.18, $0.33,0.45,0.60,0.75,0.90$ and 1.00. For the concretes and specimen geometry and dimensions of this work the overall drying times were of about 22.9 days (550 h). Finally the specimens were dried at $105^{\circ} \mathrm{C} \pm 5{ }^{\circ} \mathrm{C}$ until mass constancy, and their $m_{d}$ values recorded. These specimens cannot be used for further testing. 
The obtained data allow calculating the $A$ values of the materials under study, and to obtain the drying curves. These curves plot the variation of the water saturation, calculated through Eq. (1), with time, during drying at $50^{\circ} \mathrm{C}$; and help to obtain approximate values for the drying times needed to reach a certain $S D$ value.

\subsection{Drying step}

The specimens intended for mass transport testing are first vacuum saturated, recording their $m_{s}$ values, and their lateral surfaces sealed. Then, they are allowed to dry at $50^{\circ} \mathrm{C}$ until reaching the selected values of water saturation. By using the determined water absorption coefficients of the materials and the selected $S D$ values, it is possible to calculate, through Eq. (3), the pertinent $m_{h}$ value to be reached for each concrete specimen. Guidance on the drying times is obtained through the use of the drying curves obtained in the previous section. This lessens the experimental effort as the drying can be performed in unattended mode until reaching drying times slightly shorter than those predicted by the drying curves. Nevertheless, the final gravimetric checking is necessary for ensuring the reliability of the water saturation degrees of the specimens before mass transport testing. In this work the criterion used to set the end of drying was that the difference between the mass of the specimen and the target $m_{h}$ value was less than $0.3 \mathrm{~g}$, i.e. a difference lower than $0.06 \%$. The minimum total mass of the saturated specimens ( $3 \mathrm{~cm}$ height slices) was $575 \mathrm{~g}$; while the minimum total mass of the specimens conditioned at $S D 0.18$ was $550 \mathrm{~g}$.

\subsection{Moisture redistribution step}

The homogenization of the moisture content requires the elimination of the unidirectional moisture gradients developed into the concrete specimens during drying. This is achieved by maintaining the specimens sealed and subjected to the same temperature, $50^{\circ} \mathrm{C}$, (Parrott 1994; RILEM TC 116-PCD 1999b).

After finishing the drying step, the specimens were put into lidded polypropylene chambers of adequate geometry and dimensions to leave the minimum free space between specimen and chamber walls; and the chambers located inside the oven at $50^{\circ} \mathrm{C}$. Water vapour tightness of the chambers was checked gravimetrically during the redistribution steps. 
The redistribution times were designed according to the criterion of Parrott (1994), suggesting that the duration of the redistribution step should be at least equal to the duration of the drying period. In this work the redistribution times were set as equal to the duration of the drying period plus one day, with an absolute minimum value of 3 days. After finishing the designed redistribution periods, the closed chambers were allowed to cool slowly during three days to room temperature.

\subsection{Defining the conditions for maintaining the water saturation state during tests}

When finishing the moisture redistribution step, it is necessary to determine the environmental conditions that allow maintaining the water saturation state of the concrete specimens during the ionic diffusion tests. To this end the $R H$ within the redistribution chamber of each conditioned specimen is measured. The redistribution chambers had a top screwed stopper allowing the insertion of a hygrometer lead for measuring the $R H$ value inside the chamber. Practically constant $R H$ values were obtained typically in 90 minutes. Previous experimental evidence, (Antón 2009), demonstrated that for maintaining the target $S D$ values, the conditioned specimens must be kept inside closed containers with $R H$ values equal to those measured within the redistribution chambers, increased by $5 \%$. This way the specimens can be maintained in water vapour transfer quasi-equilibrium conditions evidenced by mass constancy.

Controlled relative humidity containers were prepared, with mixtures of glycerin and water in the appropriate proportions according to DIN (1981) to obtain each one of the quasi-equilibrium $R H$ values, and a stand for holding the concrete specimens without contact with the liquid medium. The conditioned specimens were transferred quickly from the redistribution chambers to their humiditycontrolled containers and remained in them at least for two weeks. These containers also serve to keep the specimens during the ionic diffusion tests at partial water saturation states (Climent et al. 2000 and 2002; de Vera et al. 2000 and 2007b). The constancy of the water saturation degree of the specimens during these periods was checked by periodic weighing. 


\section{Transport model}

A microscopic mass balance for the liquid phase contained in a solid porous media such as concrete leads to:

$$
\frac{\partial(\mathbf{f} s)}{\partial t}+\vec{\nabla} \cdot \vec{v}=0
$$

Where $s\left[\mathrm{~m}^{3}\right.$ water $/ \mathrm{m}^{3}$ pores $]$ is the local degree of water saturation, $\phi\left[\mathrm{m}^{3}\right.$ pores $/ \mathrm{m}^{3}$ concrete] is the porosity of concrete, $t[\mathrm{~s}]$ is time, $\vec{v}[\mathrm{~m} / \mathrm{s}]$ is the speed which moves the liquid phase and $\vec{\nabla}$. is the divergence differential operator. The liquid water flow through partially saturated porous media is usually described (Baroghel-Bouny et al. 1999 and Navarro et al. 2006) by generalizing Darcy's law:

$$
\vec{v}=-\frac{K}{\mathrm{~h}} k_{r}(s) \vec{\nabla} P
$$

Where $K\left[\mathrm{~m}^{2}\right]$ is the permeability of the saturated solid, $\eta[\mathrm{kg} /(\mathrm{m} \cdot \mathrm{s})]$ is the dynamic viscosity of liquid, $k_{r}$ [dimensionless, $0 \leq k_{r} \leq 1$ ] is the relative permeability that depends on $s, P[\mathrm{~Pa}]$ is the pressure and $\vec{\nabla}$ is the differential gradient operator. The relative permeability is related to the absorption-desorption isotherms of water. It is known that these isotherms show hysteresis, but usually the water flow model is simplified considering expressions for $k_{r}$ not taking into account hysteresis. Some authors (Navarro et al. 2006 and Savage and Janssen 1997) propose the van Genuchten (1980) formulation for concrete, so that the relative permeability can be expressed as:

$$
k_{r}(s)=\sqrt{s}\left(1-\left(1-s^{1 / m}\right)^{m}\right)^{2}(6)
$$

Where $m$ is a parameter related to the water isotherm. Whitaker (1977) showed that for materials with pores of small diameter, capillary forces are dominant and the pressure from Equation (5) is the capillary pressure $p_{c}$, which according to Luckner et al. (1989) can be expressed as:

$$
P=p_{c}=-a\left(s^{-1 / m}-1\right)^{1 / n}
$$

Where the parameters $a, m$ and $n$ are related to the water isotherm. Substituting Eqs. (5), (6) and (7) in Eq. (4) allows obtaining the transport equation: 


$$
\frac{\partial s}{\partial t}-\vec{\nabla} \cdot(D(s) \vec{\nabla} s)=0
$$

Where:

$$
D(s)=D_{0} s^{-\left(\frac{1}{m}+\frac{1}{2}\right)}\left(s^{-1 / m}-1\right)^{\frac{1}{n}-1}\left(1-\left(1-s^{1 / m}\right)^{m}\right)^{2}
$$

Being $D_{0}$ constant:

$$
D_{0}=\frac{a K}{n m \mathrm{hf}}(10)
$$

The resemblance of equation (8) with the one describing the transport in a diffusion process is the reason for naming the parameter $D(s)$ as the apparent diffusion coefficient of water.

If the parameters $D_{0}, m$ and $n$ are known and appropriate boundary conditions are applied, Equations (8) and (9) can be solved to obtain the moisture profiles $s(x, t)$.

\subsection{Obtaining the model parameters}

Model parameters $D_{0}, m$ and $n$ can be estimated from drying tests (see Section 3.1). Comparison of the experimental drying curves with the curves predicted by the model allows finding the parameter values that minimize the difference between experimental and calculated curves. The minimization is performed with the simplex optimization of Nelder-Mead algorithm (Lagarias et al. 1998). The resolution of the differential Equation (8) is made for the unidimensional case using a standard finite element method with a Crank-Nicolson time integration scheme. All calculations were performed with the MATLAB 6.1 software.

It is accepted that during drying, the relative humidity inside the oven remains constant. This constancy of the ambient relative humidity implies a constant saturation degree on the surface of the specimen. $s_{0}$ is the surface saturation degree, which is in principle unknown, as the parameters $D_{0}, m$ and $n$ are. For a given set of values of these latter four parameters, Equation (8) is solved by the finite elements method. The boundary condition applied is that the saturation degree at the surface is $s_{0}$, and the initial condition imposed is that the specimen is saturated $(s(x, 0)=1)$. The resolution of Eq. (8) provides the moisture profiles $s(x$, $t$ ) predicted with the selected values of the parameters $D_{0}, m, n$ and $s_{0}$. The main 
saturation degree of the specimen as a function of time is calculated from these profiles as:

$$
S D(t)=\frac{1}{h} \int_{0}^{h} s(x, t) d x(11)
$$

Where $h$ is the height of the specimen. The integral is calculated with the trapezoid rule. The $S D(t)$ function calculated in this way is compared to the experimental data obtained during the drying tests, by the simplex optimization algorithm, thus allowing to obtain the values of $D_{0}, m, n$ and $s_{0}$ that minimize the differences between the calculated and experimental drying curves.

\subsection{Calculation of the redistribution times}

The evolution of the moisture content profiles during the redistribution process is simulated. In this case Eq. (8) is solved using the optimized parameters determined in the preceding Section, which are applicable because the drying is performed at the same temperature as the redistribution process. The boundary condition applied is that the water flow is zero at the surface, i.e. there is no moisture exchange with the atmosphere during the redistribution step. The initial condition is that the initial moisture profile is equal to the one obtained at the end of drying. The homogeneity of the profile calculated after redistribution indicates the suitability of the selected redistribution time (see Section 3.3).

\section{Results and discussion}

Table 2 shows some measured properties of the tested concretes in the fresh state (slump), and hardened state: compressive strength, and specific gravity and voids. All results in Table 2 are mean values of three determinations. As expected, the series $\mathrm{H} 1, \mathrm{H} 2, \mathrm{H} 3$ decreases consistently the total porosity as the w/c ratio is decreased.

\subsection{Drying and conditioning experimental tests}

The drying tests data allowed obtaining the average values of the water absorption coefficients (four determinations), A, for the three concretes, shown in Table 3. The absorption capacities decrease clearly with the w/c ratio. Figure 1 shows the drying curves of the concretes, see Section 3.1. There is a linear relationship 
between $S D$ and $\sqrt{t}$ during approximately the first 200 hours, i.e. until reaching $S D$ values between $0.30-0.40$, then the moisture loss became slightly slower. The $\mathrm{H} 1$ drying curve runs over the $\mathrm{H} 2$ one in Fig. 1. This fact is due to the larger height of the $\mathrm{H} 1$ slices $(4 \mathrm{~cm})$, as compared to $\mathrm{H} 2$ and $\mathrm{H} 3$ slices $(3 \mathrm{~cm})$, see Section 2. For equal thickness of the drying concrete specimens the drying times, to reach a given $S D$ value, would be always shorter for the more porous concrete (Baroghel-Bouny et al. 1999).

On the basis of the drying curves it is possible to give approximate values for the drying times to reach the target $S D$ value for each test specimen, which are shown in Table 4. All the concrete specimens were dried at $50^{\circ} \mathrm{C}$ until reaching the $m_{h}$ values corresponding to their target $S D$, see Section 3.2. After finishing the drying step the specimens were subjected to the redistribution phase, according to the procedure described in Section 3.3. The redistribution times were set at the values indicated in Table 5 (second to fourth columns).

Once finished the water redistribution step, and allowed the specimens, into their closed chambers, to cool down to room temperature, the relative humidity inside the redistribution chambers was measured, according to Section 3.4. Fig. 2 shows a typical plot of the recordings performed for such measurements. The averaged measured $R H$ value, for each concrete type and water saturation degree, was increased by 5\%, see Section 3.4. Table 6 contains the modified $R H$ values for each concrete and water saturation degree. These latter values were used to prepare the controlled relative humidity chambers, (containing the appropriate glycerin-water mixtures), for storing the preconditioned concrete specimens to be used in diffusion tests at partial water saturation states. The water saturated specimens were stored in closed containers with pure water.

\subsection{Water transport model calculations}

The calculations were performed by fitting the experimental drying data (symbols in Fig. 1), into the transport model, see Section 4.1. The fitting parameters, together with the correlation coefficients, can be found in Table 7. Lines in Fig. 1 represent the calculated drying curves, showing a good agreement between experimental and calculated data.

The relative permeabilities $k_{r}(s)$ and apparent diffusion coefficients of water $D(s)$, which are obtained by applying the abovementioned parameters to Equations (6) 
and (9), for each of the concretes studied, are shown as a function of $s$ in Fig. 3 and Fig. 4, respectively. It is appreciable from Fig. 3 that, for the three concretes, the relative permeability decreases very fast when the saturation degree decreases, in concordance with results of other authors (Baroghel-Bouny et al. 1999; Monlouis-Bonnaire et al. 2004). It is also noted that the permeability decreases with the porosity of concrete.

The values of the apparent diffusion coefficient of water are of the same order of magnitude as those found by Crausse et al. (1996) on cement pastes and concretes; and they show maxima located between 0.85 and $0.95 s$, see Fig. 4 . The diffusion coefficient decreases rapidly to zero when the saturation degree is close to 1 or to 0 . When $s$ increases from 0.10 to 0.90 , the diffusion coefficient grows smoothly.

Fig. 5 shows the profiles of the local saturation degree, calculated with the model at the end of the drying step of the specimens. Other authors have reported experimental moisture profiles in drying tests, measured by the gamma-rays attenuation technique (Baroghel-Bouny et al. 1999), obtaining morphologically similar profiles to those presented here. For better comparison, the depth $x$ is divided by the height of the specimen $h$, because the specimens are of different sizes. These are also the initial profiles for the moisture redistribution phases. It is noted that in all cases there are significant moisture content gradients, in agreement with observations of other authors (Parrott 1994). The local saturation degree, $s$, is close to zero at the surface, see also $s_{0}$ values in Table 7, while $s$ is higher than the main saturation degree in the central part of the specimen. These strong gradients put again in evidence the need for a redistribution phase to obtain a homogeneously distributed saturation degree. For the case of the main saturation degree of 0.90 , drying has only affected about half a $\mathrm{cm}$ from the concrete surface, while the rest of the sample remains almost water saturated. The centre of the specimen is affected by the drying for main saturation degrees below 0.75 . The three concretes studied show rather similar behaviour. However, Fig. 5 shows that the larger is the concrete porosity the higher gradients are generated. Nevertheless, it must be taken into account that the different height of the tested specimens, see Section 2, can lead to steeper variations of $s$ for $\mathrm{H} 1$, as compared to $\mathrm{H} 2$ and $\mathrm{H} 3$ concretes, in Fig. 5. The most important differences between the tested concretes are observed for the main $S D$ values of 0.60 and 0.45 . 
Fig. 6 shows an example of the results of applying the model to calculate the evolution with time of the local saturation degree profile for $\mathrm{H} 1$ concrete, during the moisture redistribution phase with a 0.60 main $S D$ value. It can be seen that water is redistributed, increasing rapidly the local saturation degree near the surface and lowering it at the center of the specimen. For long times a homogeneous profile is reached, with local saturation degree values equal to the main saturation degree throughout the whole specimen. To quantify the homogeneity of the profiles we calculate the difference between the maximum value (center of the specimen) and the minimum value (surface) of $s$, as a function of the redistribution time, using Equation (12):

$$
\Delta s(t)=s(h / 2, t)-s(0, t)
$$

A homogeneous distribution is obtained when $\Delta s=0$, however this will happen for long periods. For practical purposes the redistribution time may be considered adequate if $\Delta s$ is below an arbitrary threshold value. Table 5 (fifth to seventh columns) shows the calculated values of $\Delta s$ for the applied redistribution times (second to fourth columns of Table 5), indicating acceptable homogeneities of the moisture profiles for the selected redistribution times, if we accept as correct the model calculations. It must be recalled that the criterion for setting the redistribution times was based on previous recommendations (Parrott 1994), and that it was intended to represent a compromise between the homogeneity of the resulting profile and the time required for conditioning the specimens. H2 concrete shows a greater homogeneity, i.e. lower values of $\Delta s$, while $\mathrm{H} 1$ has the least homogeneous water distributions, with $\mathrm{H} 3$ concrete showing intermediate values. These differences are due to two factors: transport of water is more difficult the lower is the porosity of concrete ( $\mathrm{H} 2$ profiles more homogeneous than $\mathrm{H} 3$ ones); and an increased thickness of the specimen also makes more difficult the full redistribution ( $\mathrm{H} 2$ more homogeneous than $\mathrm{H} 1)$. The heterogeneity of the profiles is maximal for a 0.60 saturation degree and decreases rapidly when $S D$ decreases. It is necessary to stress that the moisture profiles presented in this work are only model calculations, without checking against experimental measurements, which will be the object of future research.

The transport model also offers the possibility to calculate redistribution times for ensuring that the calculated $\Delta s$ remains lower than an arbitrarily chosen value. The eighth to tenth columns of Table 5 show the calculated redistribution times 
for the concrete specimens studied in this work, with the condition that $\Delta s \leq 0.01$ $(1 \%)$.

On the basis of the calculated redistribution times (last columns of Table 5), and for the cases where the model calculations are not feasible, a different criterion for choosing the redistribution times (see Section 3.3), for concrete types and specimen dimensions similar to those of this work, can be formulated: the redistribution times must be set as equal to the duration of the drying period plus one day, with an absolute minimum value of 7 days.

\subsection{Maintenance of the hygric states of concrete after conditioning}

The strategy chosen in this protocol to maintain the well characterized and homogeneously distributed water saturation states of concrete after conditioning, was to keep the specimens into appropriate controlled $R H$ containers, whose values were based on those measured at the end of the redistribution steps, see Sections 3.4 and 5.1 and Table 6 (third column). Nevertheless, further gravimetric checking of the $S D$ values of each specimen was maintained during periods ranging from one to nine months after putting the specimens into their controlled $R H$ containers and further diffusion tests (Antón 2009). Some small deviations of the target $S D$ values were observed only during the first two weeks, all of them by drying. If necessary, the $R H$ of the environment was again modified, by changing slightly the concentration of the glycerin-water mixtures, until observing constancy of the target $S D$ values. The final recorded $R H$ values are found in the fourth column of Table 6 . 


\section{Conclusion}

On the basis of the results of this work, it may be stated that the proposed conditioning procedure allows to install into the concrete specimens any selected value of the water saturation degree with homogeneous distribution. The protocol has the following steps: obtaining basic drying data at $50^{\circ} \mathrm{C}$ (water absorption capacity and drying curves); unidirectional drying of the specimens at $50^{\circ} \mathrm{C}$ until reaching the target $S D$ values; redistribution phase in closed containers at $50^{\circ} \mathrm{C}$ (with measurement of the quasi-equilibrium $R H$ ); storage into controlled $R H$ chambers until and during mass transport tests, if necessary. A water transport model has been used to derive transport parameters of the tested materials from the drying data, i.e. relative permeabilities and apparent water diffusion coefficients. The model also allows calculating moisture profiles during isothermal drying and redistribution phases, thus allowing optimization of the redistribution times for obtaining homogeneous moisture distributions. Nevertheless, for the cases where the model calculations are not feasible, simple guidance rules can be given about the redistribution times. For concrete types and specimen dimensions similar to those of this work, the redistribution times should be set as equal to the duration of the drying period plus one day, with an absolute minimum value of 7 days. The redistribution times will always be dependent on the concrete characteristics and on the specimen dimensions.

\section{Acknowledgements}

We thank the funding received for this research from the Ministerio de Ciencia e Innovación and Ministerio de Economía y Competitividad of Spain and Fondo Europeo de Desarrollo Regional (FEDER) through project BIA2010-20548, and from the Ministerio de Fomento of Spain through project C34/2006. Authors would like to thank Holcim España, S.A. for providing the cement and aggregates, and Sika S.A.U. España for the superplasticizer, needed for this research. 


\section{References}

AENOR (1984) Ensayos de hormigón. Rotura por compresión (Concrete tests. Compressive strength test). UNE 83304:1984. Asociación Española de Normalización y Certificación, Madrid, Spain. (Only available in Spanish)

AENOR (1990) Ensayos de hormigón. Medida de la consistencia del hormigón fresco. Método del cono de Abrams (Concrete tests. Measurement of fresh concrete consistency. Abrams' cone method). UNE 83313:1990. Asociación Española de Normalización y Certificación, Madrid, Spain. (Only available in Spanish)

Antón C (2009) Influencia del contenido de humedad del hormigón sobre la difusividad del ión cloruro (Influence of the moisture content of concrete on the chloride ion diffusivity). PhD Thesis, Universidad Autónoma de Madrid, Spain. (Only available in Spanish)

ASTM (1994) Standard test method for specific gravity, absorption and voids in hardened concrete. C642-90. American Society for Testing and Materials, Philadelphia, USA

ASTM (1997) Standard test method for electrical indication of concrete's ability to resist chloride ion penetration. C1202-97. American Society for Testing and Materials, Philadelphia, USA

Baroghel-Bouny V, Mainguy M, Lassabatere T, Coussy O (1999) Characterization and identification of equilibrium and transfer moisture properties for ordinary and high-performance cementitious materials. Cem Conc Res 29:1225-1238

Carcassès M, Abbas A, Ollivier J-P, Verdier J (2002) An optimised preconditioning procedure for gas permeability measurement. Mater Struct 35:22-27

CEN/TC 104 (2009) Testing hardened concrete - Determination of the chloride resistance of concrete, unidirectional diffusion. Final draft prCEN/TS 12390-11

Climent MA, de Vera G, López JF, García C, Andrade C (2000) Transport of chlorides through non-saturated concrete after an initial limited chloride supply. In: Andrade C, Kropp J (eds) Proc. of the $2^{\text {nd }}$ int. workshop on testing and modelling the chloride ingress into concrete, RILEM Publications, Cachan, France, pp 173-187

Climent MA, de Vera G, López JF, Viqueira E, Andrade C (2002) A test method for measuring chloride diffusion coefficients through nonsaturated concrete. Part I. The instantaneous plane source diffusion case. Cem Conc Res 32 (7):1113-1123 
Crausse P, Laurent JP, Perrin B (1996) Influence des phénomènes d'hystérésis sur les propriétés hydriques de matériaux poreux. Rev Gén Therm 35:95-106

de Vera G (2000) Ingreso de cloruros en hormigón. Métodos de análisis, detección no destructiva y modelización del transporte tras un aporte inicial limitado (Chloride ingress in concrete. Analytical methods, non destructive detection and modelling of transport after an initial limited supply). PhD Thesis, Universitat d'Alacant. (Only available in Spanish)

de Vera G, Climent MA, Antón C, Sánchez I, Andrade C (2007a) Chloride diffusion coefficients through concrete partially submerged and partially exposed to the atmosphere. In: Ferreira RM, Gulikers J, Andrade C (eds.) Proc. of the int. RILEM workshop on integral service life modelling of concrete structures. RILEM Publications (PRO-56), Cachan, France, pp 23-30

de Vera G, Climent MA, Viqueira E, Antón C, Andrade C (2007b) A test method for measuring chloride diffusion coefficients through partially saturated concrete. Part II. The instantaneous plane source diffusion case with chloride binding consideration. Cem Conc Res 37 (5):714-724

DIN (1981) Standard atmospheres over aqueous solutions. Saturated salt solutions, glycerol solutions (50 008 Part 1). Deutsches Institut für Normung (DIN), Berlin

Guimarães ATC (2000) Vida útil de estruturas de concreto armado em ambientes marítimos (Service life of reinforced concrete structures in maritime environments). PhD Thesis, Universidade de São Paulo - PCC/USP, São Paulo, Brasil. (Only available in Portuguese)

Guimarães ATC, Helene PRL (2004) Chloride diffusion and the influence of the saturation degree of the concrete. In: Andrade C, Kropp J (eds) Proc. of the $3^{\text {rd }}$ int. workshop on testing and modelling the chloride ingress into concrete. RILEM Publications, Cachan, France, pp 237-255

Guimarães ATC, Helene PRL (2005) Diffusion of chloride ions in unsaturated concrete: Forecast of service life in a wet-dry environment. ACI SP 229-12, Farmington Hills, MI, USA, 175-193

Guimarães ATC, Climent MA, de Vera G, Vicente FJ, Rodrigues FT, Andrade C (2011) Determination of chloride diffusivity through partially saturated Portland cement concrete by a simplified procedure. Const Build Mat 25 (2):785-790

Hilsdorf HK (1995) Introduction and problem statement. In: Kropp J, Hilsforf HK (eds) Performance criteria for concrete durability, E \& FN Spon, London, pp 1-3

Kropp J, Hilsdorf HK, Grube H, Andrade C, Nilsson L-O (1995) Transport mechanisms and definitions. In: Kropp J, Hilsforf HK (eds) Performance criteria for concrete durability, E \& FN Spon, London, pp 4-14 
Lagarias JC, Reeds JA, Wright MH, Wright PE (1998) Convergence properties of the NelderMead simplex method in low dimensions. SIAM Journal of Optimization 9:112-147

Luckner L, van Genuchten MT, Nielsen DR (1989) A consistent set of parametric models for the two-phase flow of inmiscible fluids in the subsurface gaseous phase. Water Resour Res 25:21872193

McGrath PF, Hooton RD (1999) Re-evaluation of the AASHTO T259 90-day salt ponding test. Cem Conc Res 29:1239-1248

Monlouis-Bonnaire JP, Verdier J, Perrin B (2004) Prediction of the relative permeability to gas flow of cement-based materials. Cem Conc Res 34:737-744

MPR 2008 Instrucción para la recepción de cementos (RC-08). Ministerio de la Presidencia (MPR) of Spain, Madrid. (Only available in Spanish). (Equivalent to the european standard EN 197-1)

Navarro V, Yustres A, Cea L, Candel M, Juncosa R, Delgado J (2006) Characterization of the water flow through concrete based on parameter estimation from infiltration tests. Cem Conc Res 36:1575-1582

Nielsen EP, Geiker MR (2003) Chloride diffusion in partially saturated cementitious material. Cem Conc Res 33 (1):133-138

Nokken MR, Hooton RD (2002) Dependence of rate of absorption on degree of saturation of concrete. ASTM Cem Conc Agg 24 (1):20-24

Ollivier J-P, Massat M, Parrott LJ (1995) Parameters influencing transport characteristics. In: Kropp J, Hilsforf HK (eds) Performance criteria for concrete durability, E \& FN Spon, London, pp 33-96

Parrott LJ, Chen ZH (1990) Some factors influencing air permeation measurements in cover concrete. Mater Struct 24 (144):403-408

Parrott LJ (1994) Moisture conditioning and transport-properties of concrete test specimens. Mater Struct 27 (172):460-468

RILEM TC 116-PCD (1999a) Final Report: Concrete durability - An approach towards performance testing. Mater Struct 32:163-173 
RILEM TC 116-PCD (1999b) Recommendations: A. Preconditioning of concrete test specimens for the measurement of gas permeability and capillary absorption of water. Mater Struct 32:174176

Romer M (2005) Effect of moisture and concrete composition on the Torrent permeability measurement. Mater Struct 38:541-547

Safiuddin M, Hearn N (2005) Comparison of ASTM saturation techniques for measuring the permeable porosity of concrete. Cem Conc Res 35:1008-1013

Savage BM, Janssen DJ (1997) Soil physics principles validated for use in predicting unsaturated moisture movement in Portland cement concrete. ACI Mater J 94:63-70

van Genuchten MT (1980) A closed-form equation for predicting the hydraulic conductivity of unsaturated soils. Soil Sci Soc Am J 44:892-898.

Whitaker S (1977) Simultaneous heat, mass, and momentum transfer in porous media: a theory of drying. Adv Heat Transf 13:119-203. 


\section{FIGURE CAPTIONS}

Figure 1. Experimental (symbols) and calculated (lines) drying curves. The calculations were performed by fitting the experimental data into the transport model.

Figure 2. Determination of the relative humidity inside the water redistribution chambers of three samples of concrete $\mathrm{H} 1$ with saturation degree of 0.75 .

Figure 3. Dependence of the relative permeabilities of the concretes studied on the water saturation degree.

Figure 4. Dependence of the apparent water diffusion coefficients of the concretes studied on the water saturation degree.

Figure 5. Profiles of the local saturation degree, calculated at the end of the drying step, for the main saturation degree (SD) of $0.90,0.60$ and 0.33 (A) and $0.75,0.45$ and 0.18 (B).

Figure 6. Temporal evolution of the local saturation degree profile during the redistribution phase of concrete $\mathrm{H} 1$ with a target main saturation degree of 0.60 . 


\section{TABLES}

Table 1. Dosages of the concrete mixes.

\begin{tabular}{|c|c|c|c|}
\hline & H1 & H2 & H3 \\
\hline Cement $\left(\mathrm{Kg} / \mathrm{m}^{3}\right)$ & 350 & 350 & 450 \\
\hline Sand $\left(\mathrm{Kg} / \mathrm{m}^{3}\right)$ & 630.3 & 662.8 & 628 \\
\hline $\begin{array}{c}\text { Gravel } 4-6 \mathrm{~mm} \\
\left(\mathrm{Kg} / \mathrm{m}^{3}\right)\end{array}$ & 465.5 & 489.5 & 637 \\
\hline $\begin{array}{c}\text { Gravel } 6-12 \mathrm{~mm} \\
\left(\mathrm{Kg} / \mathrm{m}^{3}\right)\end{array}$ & 679 & 714 & 0.38 \\
\hline $\mathrm{w} / \mathrm{c}$ & 0.60 & 0.50 & 1.5 \\
\hline $\begin{array}{c}\text { Superplasticizer }(\% \\
\text { ref. to cem. mass })\end{array}$ & 0.4 & 1.4 & \\
\hline
\end{tabular}

Table 2. Properties of the tested concretes.

\begin{tabular}{|l|c|c|c|}
\hline \multicolumn{1}{|c|}{ Concrete } & H1 & H2 & H3 \\
\hline Abrams' slump cone (cm) & 15 & 17.5 & 20 \\
\hline Average compressive strength (MPa) & 31.3 & 40.3 & 40.2 \\
\hline Bulk specific gravity, dry (Kg/m $\left.{ }^{3}\right)$ & 2.18 & 2.23 & 2.29 \\
\hline Volume of permeable pore space (voids) (\%) & 15.6 & 12.6 & 10.5 \\
\hline
\end{tabular}

Table 3. Mean values (4 determinations) of the water absorption coefficient of the studied concretes.

\begin{tabular}{|c|c|c|}
\cline { 2 - 3 } \multicolumn{1}{c|}{} & \multicolumn{2}{c|}{ Absorption coefficient $A$} \\
\cline { 2 - 3 } \multicolumn{1}{c|}{} & Average & Standard deviation \\
\hline H1 & 0.0750 & 0.0028 \\
\hline H2 & 0.0571 & 0.0024 \\
\hline H3 & 0.0554 & 0.0016 \\
\hline
\end{tabular}


Table 4. Approximate drying times at $50{ }^{\circ} \mathrm{C}$ for each concrete and target saturation degree.

\begin{tabular}{|c|c|c|c|}
\cline { 2 - 4 } \multicolumn{1}{c|}{} & \multicolumn{3}{c|}{ Approximate drying times (days) } \\
\hline$S D$ & $\mathrm{H} 1$ & $\mathrm{H} 2$ & $\mathrm{H} 3$ \\
\hline 1.00 & 0 & 0 & 0 \\
\hline 0.90 & 0.15 & 0.13 & 0.19 \\
\hline 0.75 & 1.0 & 0.83 & 1.3 \\
\hline 0.60 & 2.6 & 2.3 & 3.5 \\
\hline 0.45 & 5.1 & 4.4 & 7.0 \\
\hline 0.33 & 8.1 & 7.3 & 11.6 \\
\hline 0.18 & 15.1 & 14.8 & 23.9 \\
\hline
\end{tabular}

Table 5. Water redistribution phases in closed chambers at $50^{\circ} \mathrm{C}$. Applied redistribution times and calculated data regarding homogeneity of the distributions.

\begin{tabular}{|c|c|c|c|c|c|c|c|c|c|}
\cline { 2 - 11 } \multicolumn{1}{c|}{} & \multicolumn{3}{|c|}{$\begin{array}{c}\text { Applied } \\
\text { redistribution } \\
\text { times (days) }\end{array}$} & \multicolumn{4}{c|}{$\Delta s$} & \multicolumn{3}{c|}{$\begin{array}{c}\text { Calculated redistribution } \\
\text { times (days) for } \Delta s \leq \\
0.01\end{array}$} \\
\hline$S D$ & $\mathrm{H} 1$ & $\mathrm{H} 2$ & $\mathrm{H} 3$ & $\mathrm{H} 1$ & $\mathrm{H} 2$ & $\mathrm{H} 3$ & $\mathrm{H} 1$ & $\mathrm{H} 2$ & $\mathrm{H} 3$ \\
\hline 1.00 & 0 & 0 & 0 & - & - & - & 0 & 0 & 0 \\
\hline 0.90 & 3 & 3 & 3 & 0.0455 & 0.0148 & 0.0393 & 5.2 & 3.4 & 4.9 \\
\hline 0.75 & 3 & 3 & 3 & 0.0886 & 0.0349 & 0.0856 & 6.3 & 4.3 & 6.3 \\
\hline 0.60 & 4 & 4 & 4 & 0.0520 & 0.0198 & 0.0582 & 6.7 & 4.8 & 7.2 \\
\hline 0.45 & 6 & 5 & 8 & 0.0166 & 0.0130 & 0.0109 & 6.9 & 5.4 & 8.2 \\
\hline 0.33 & 10 & 9 & 14 & 0.0024 & 0.0018 & 0.0018 & 7.1 & 6.0 & 9.3 \\
\hline 0.18 & 15 & 14 & 23 & 0.0005 & 0.0007 & 0.0007 & 7.4 & 7.2 & 11.7 \\
\hline
\end{tabular}


Table 6. Relative humidities of controlled environment chambers for each concrete and saturation degree, as determined at the end of the redistribution phase with the modifications described in Section 3.4, and final values.

\begin{tabular}{|c|c|c|c|}
\hline$S D$ & Concrete & $\begin{array}{c}R H(\%) \\
\text { (end of redistribution phase) }\end{array}$ & $\begin{array}{c}\text { RH (\%) } \\
\text { (final values) }\end{array}$ \\
\hline \multirow{3}{*}{0.90} & H1 & 98 & 98 \\
\hline & $\mathrm{H} 2$ & 98 & 98 \\
\hline & H3 & 98 & 98 \\
\hline \multirow{3}{*}{0.75} & H1 & 90 & 90 \\
\hline & $\mathrm{H} 2$ & 85 & 87 \\
\hline & H3 & 80 & 85 \\
\hline \multirow{3}{*}{0.60} & H1 & 80 & 80 \\
\hline & $\mathrm{H} 2$ & 75 & 75 \\
\hline & H3 & 70 & 70 \\
\hline \multirow{3}{*}{0.45} & H1 & 70 & 70 \\
\hline & $\mathrm{H} 2$ & 70 & 70 \\
\hline & H3 & 62 & 65 \\
\hline \multirow{2}{*}{0.33} & H1 & 62 & 65 \\
\hline & $\mathrm{H} 2$ & 62 & 65 \\
\hline
\end{tabular}

Table 7. Fitting parameters of the experimental drying data into the transport model.

\begin{tabular}{|l|r|c|c|c|c|}
\hline Concrete & $\begin{array}{c}D_{0} \\
\left(10^{-8} \mathrm{~m}^{2} / \mathrm{s}\right)\end{array}$ & $m$ & $n$ & $s_{0}$ & $r$ \\
\hline H1 & 1.7594 & 0.1410 & 0.5324 & $1.72 \cdot 10^{-4}$ & 0.9994 \\
\hline H2 & 2.2487 & 0.1158 & 0.5348 & $2.50 \cdot 10^{-5}$ & 0.9993 \\
\hline H3 & 1.7965 & 0.1079 & 0.5347 & $9.92 \cdot 10^{-6}$ & 0.9996 \\
\hline
\end{tabular}




\section{FIGURES}

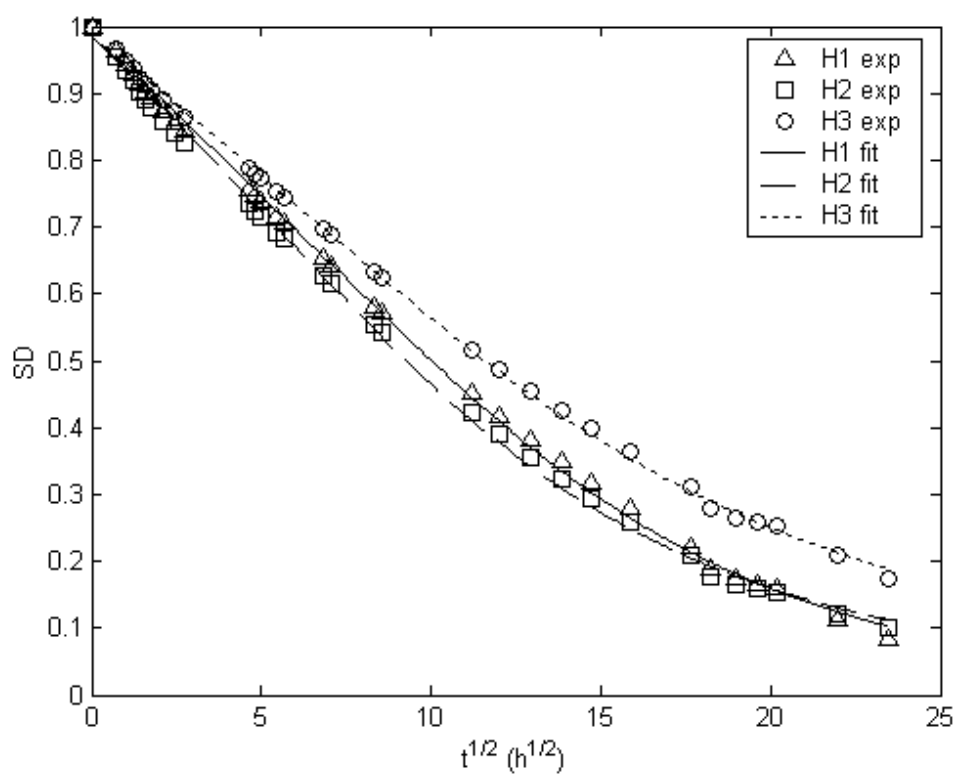

Figure 1. Experimental (symbols) and calculated (lines) drying curves. The calculations were performed by fitting the experimental data into the transport model.

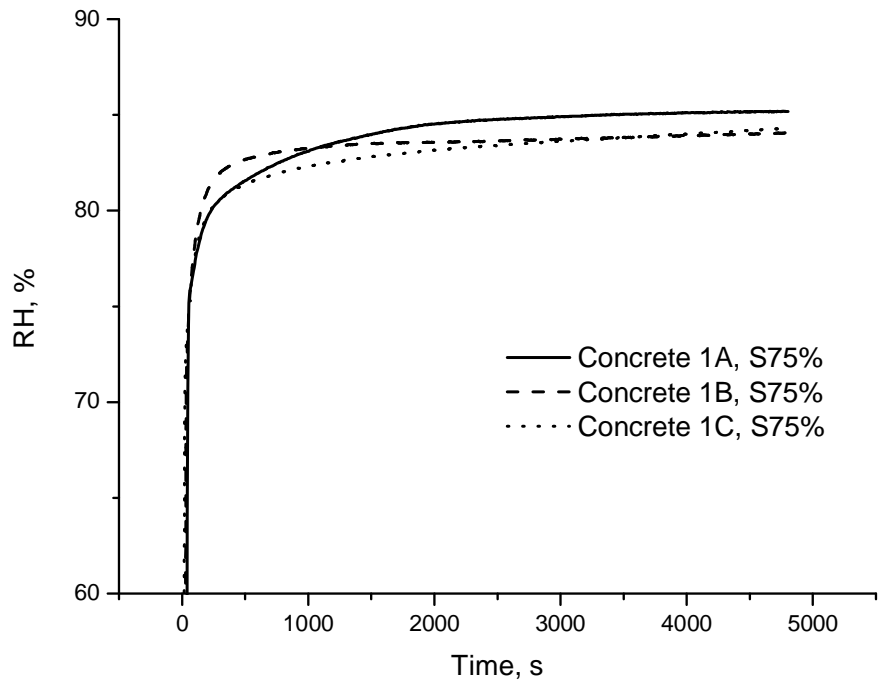

Figure 2. Determination of the relative humidity inside the water redistribution chambers of three samples of concrete $\mathrm{H} 1$ with saturation degree of 0.75 . 


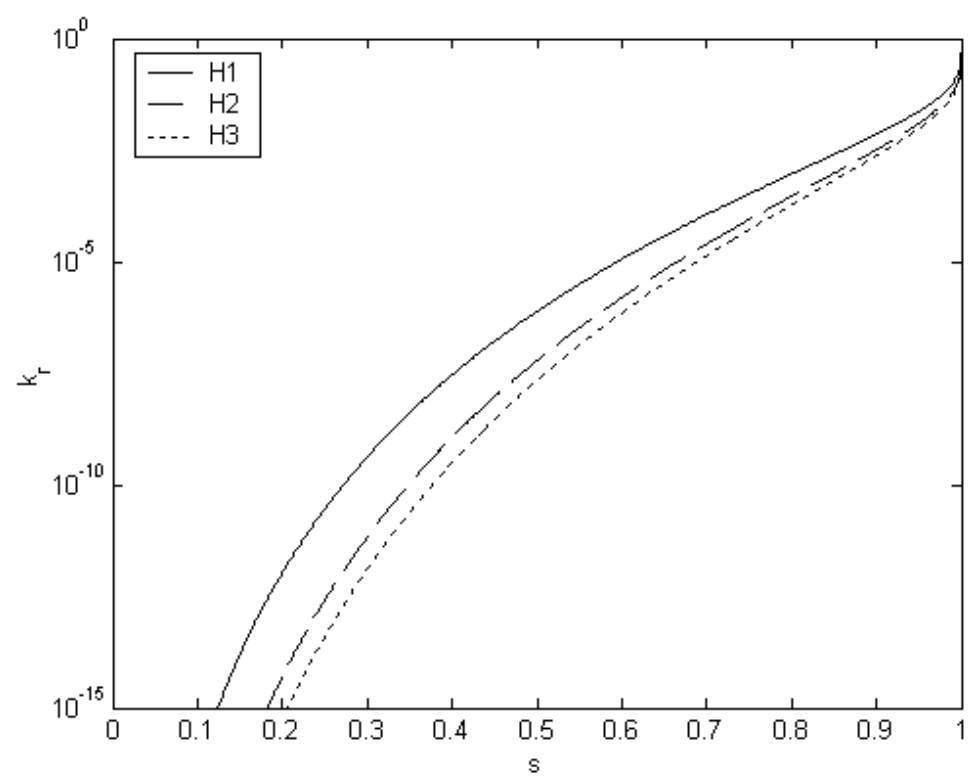

Figure 3. Dependence of the relative permeabilities of the concretes studied on the water saturation degree.

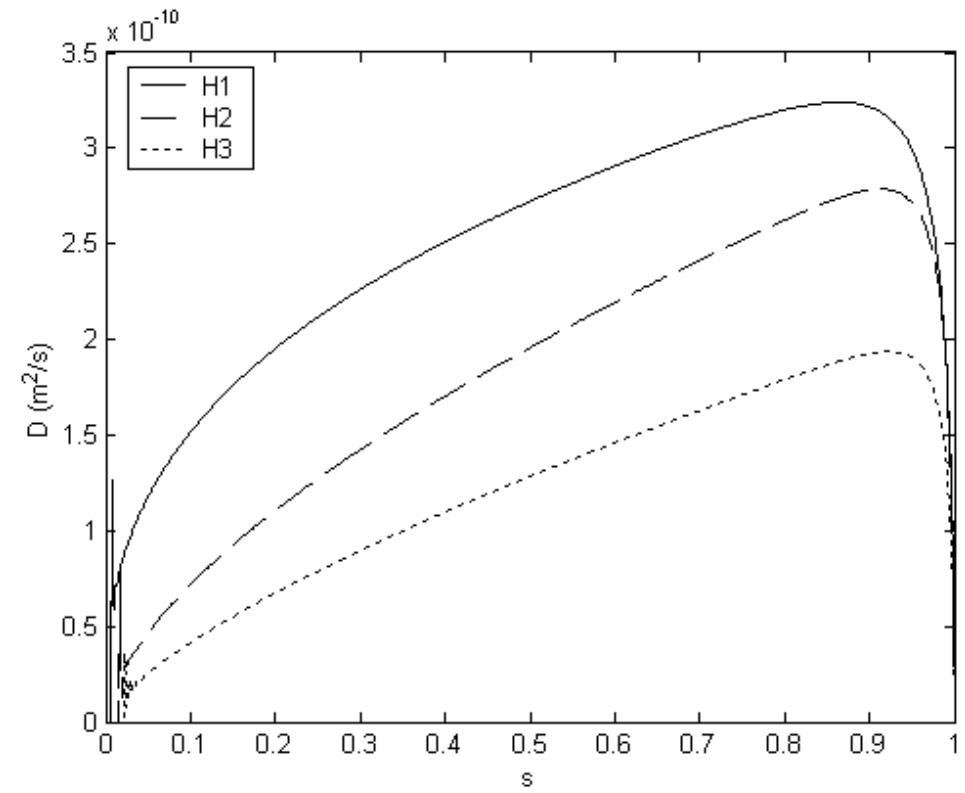

Figure 4. Dependence of the apparent water diffusion coefficients of the concretes studied on the water saturation degree. 

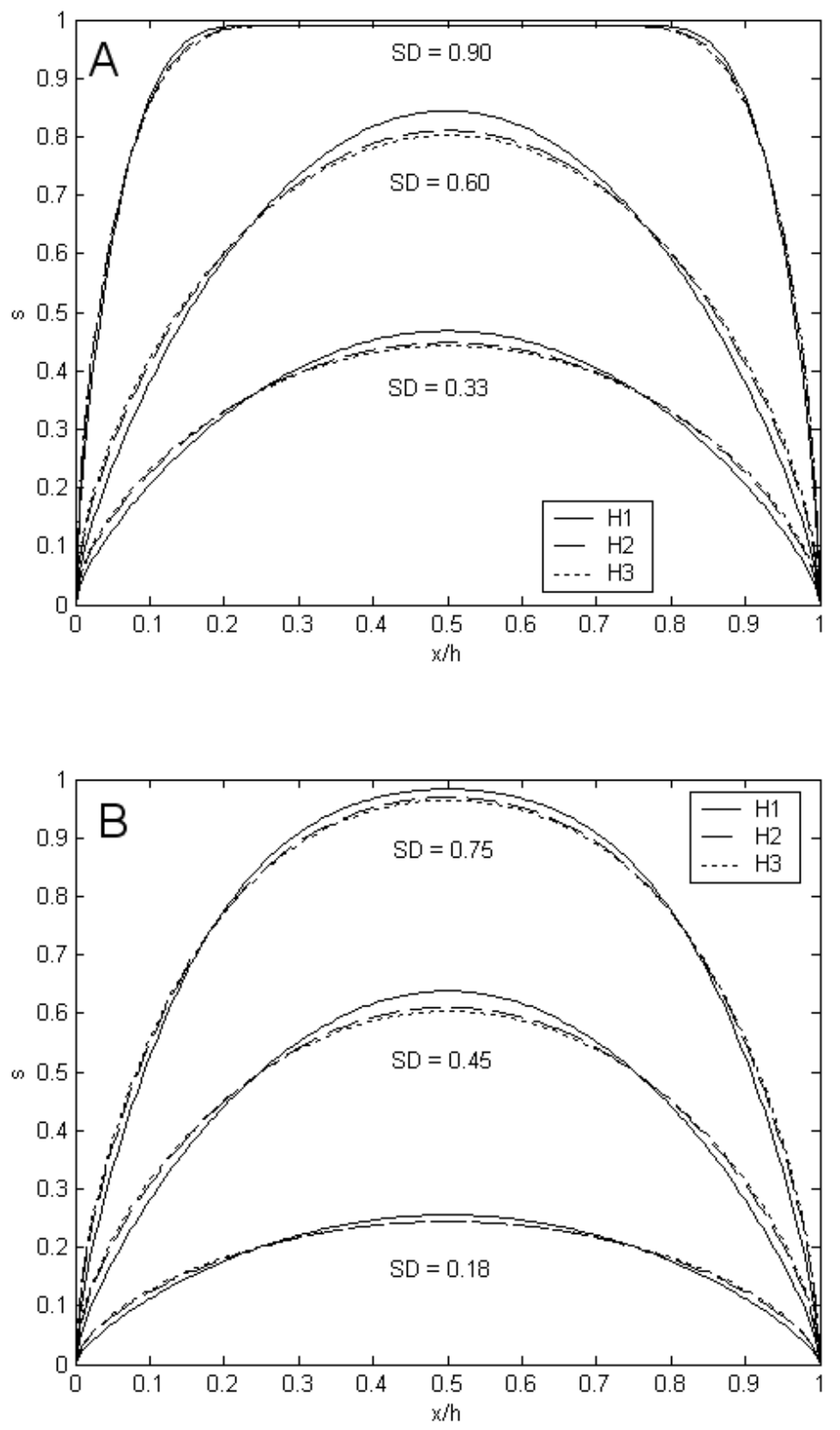

Figure 5. Profiles of the local saturation degree, calculated at the end of the drying step, for the main saturation degree (SD) of 0.90, 0.60 and 0.33 (A) and $0.75,0.45$ and 0.18 (B). 


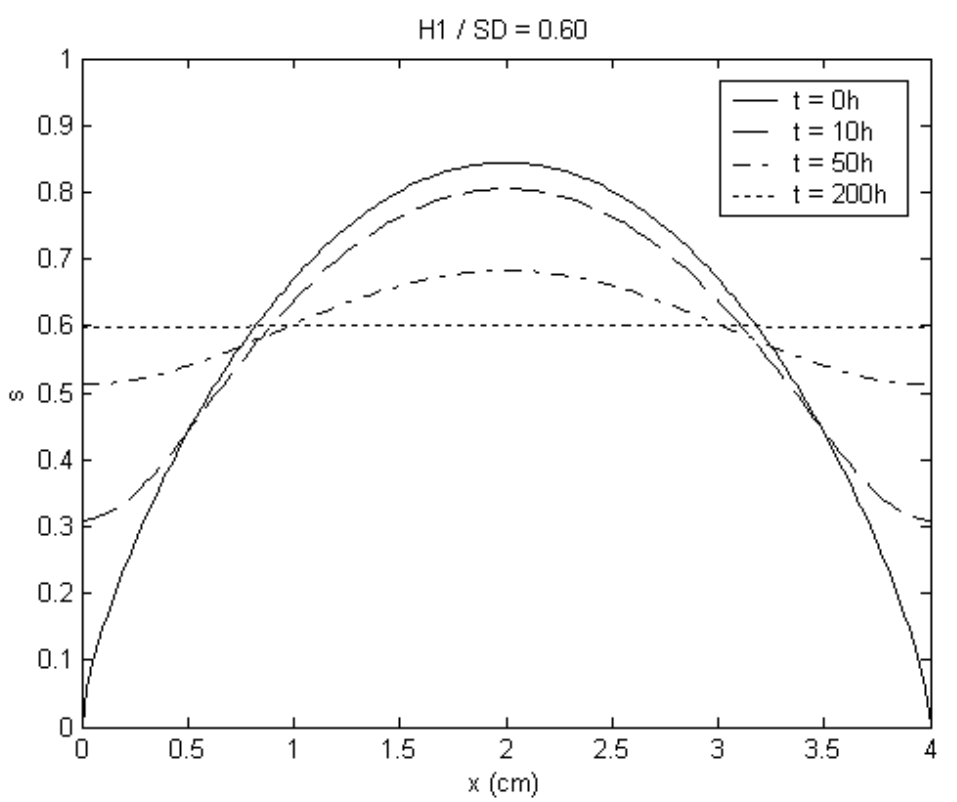

Figure 6. Temporal evolution of the local saturation degree profile during the redistribution phase of concrete $\mathrm{H} 1$ with a target main saturation degree of 0.60 . 